\title{
PEDIATRIC UROLOGY
}

\section{Outcome analysis of severe chordee correction using tunica vaginalis as a flap in boys with proximal hypospadias}

Braga LH, Pippi Salle JL, Dave S, Bagli DJ, Lorenzo AJ, Khoury AE

Division of Urology, Hospital for Sick Children, Toronto, Ontario, Canada

J Urol. 2007; 178: 1693-7; discussion 1697

Purpose: There is ongoing controversy regarding optimal treatment for severe ventral curvature. It has been suggested that ventral corporeal lengthening may be associated with recurrent curvature and erectile dysfunction. To further assess these issues we reviewed our experience with ventral penile lengthening for correcting the severe ventral curvature associated with proximal hypospadias.

Materials and Methods: We reviewed the records of 38 boys with severe hypospadias and congenital ventral curvature greater than 45 degrees who were treated at our institution from 1995 to 2004 with placement of a flap or graft in the corporeal bodies to straighten the phallus. Of the patients 21 had perineal and 17 had penoscrotal hypospadias, including 22 with associated penoscrotal transposition and/or bifid scrotum and 6 with ambiguous genitalia. Testosterone stimulation before surgery was given in 11 children at surgeon discretion.

Results: Median age at surgery was 15 months. The urethral plate was divided in $94.7 \%$ of patients. A tunica vaginalis flap was used alone in 23 cases and associated with dura, pericardium or small intestinal submucosa in 8,2 and 1, respectively. The remaining 4 patients underwent ventral grafting alone, including lyophilized dura in 1, pericardium in 1 and dermis in 1 . Urethral reconstruction was achieved by the transverse island flap technique or 1 of its modifications in 34 children. Four boys underwent a 2-stage procedure. Followup available on 35 of 38 patients was 1 to 11 years (median 5.3). Recurrent ventral curvature in 5 of 35 patients was mild in 1 and clinically significant, requiring re-intervention, in 4 . Four of 9 patients $(44.4 \%)$ who underwent corporeal grafting with lyophilized dura had recurrent ventral curvature vs. 1 of $23(4.3 \%)$ who had a tunica vaginalis 
flap (chi-square 5.14, $\mathrm{p}=0.02$ ). At last followup straight erections were documented by patients and/or parents in 30 of 35 children (85.7\%). Conclusions: The short-term outcome of ventral penile lengthening using tunica vaginalis flap alone for correcting severe chordee is favorable with a 95\% success rate. Dural grafts were associated with a higher risk of recurrent ventral curvature compared to tunica vaginalis flaps. Although most of our patients were not yet adults, when chordee and erectile dysfunction may become apparent, we believe that tunica vaginalis flap repair is a good option for correcting severe ventral curvature.

\section{Editorial Comment}

Important points made in the manuscript include that if grafting is the surgeon's choice, that the grafts should be $20-30 \%$ larger than the defect. The tunica vaginalis flap was easy to harvest and these authors had excellent success. Being a flap rather than a graft, it can be cut to the appropriate size. The blood supply has been shown to be reliable and the complications noted doing a one-stage repair are in line with what one would expect from one-stage repairs without the severe curvature correction. I find most mild chordee can be corrected dorsally but I agree with these authors that the tunica vaginalis flap is their procedure of choice to correct severe chordee on the ventral aspect of the penis.

Dr. Brent W. Snow

Division of Urology

University of Utah Health Sci Ctr

Salt Lake City, Utah, USA

E-mail:brent.snow@hsc.utah.edu

\section{Long-term tolerability of tolterodine extended release in children 5-11 years of age: results from a 12-month, open-label study}

Nijman RJ, Borgstein NG, Ellsworth P, Siggaard C

University Medical Centre Groningen, Groningen, The Netherlands

Eur Urol. 2007; 52: 1511-6

Objective: To evaluate the long-term tolerability of tolterodine extended release (ER) in children (aged 5-11 yr) with urgency urinary incontinence (UUI).

Methods: This was a multicenter, open-label extension of a 12-wk, double-blind, placebo-controlled study of tolterodine ER. Patients had UUI suggestive of detrusor overactivity $(>/=1$ diurnal incontinence episode per $24 \mathrm{~h}$ for $>/=5$ of $7 \mathrm{~d}$ ) and $>/=6$ voids per $24 \mathrm{~h}$ at baseline and had completed the 12-wk double-blind study. Patients received tolterodine ER ( $2 \mathrm{mg}$ once daily) for $12 \mathrm{mo}$. The primary end points were the incidence and severity of adverse events (AEs) and the incidence and reasons for withdrawals. Visits were scheduled at 3, 6, 9, and $12 \mathrm{mo}$, and investigators were instructed to report all AEs. At 6 and 12 mo, vital signs were recorded and a physical examination was performed.

Results: A total of 318 patients were enrolled (double-blind tolterodine ER, $n=221$; placebo, $n=97$ ). The majority of patients were white (90\%), mean $+/$ - SD age was $7.6+/-1.5 \mathrm{yr}$, and 54\% were boys. Forty-nine percent of patients reported $>/=1 \mathrm{AE}$ during the study, similar to that observed in the preceding 12 -wk study $(42 \%)$. The most frequent AEs were urinary tract infection (7\%), nasopharyngitis $(5 \%)$, headache (5\%), and abdominal pain (4\%); 111 (35\%) patients withdrew. The most common reasons for withdrawal were lack of efficacy (12\%), symptom improvement (8\%), and withdrawn consent (6\%). Ten patients $(3 \%)$ withdrew because of AEs. Conclusion: Long-term treatment with tolterodine ER was well tolerated in children with UUI. 


\section{Urological Survey}

\section{Editorial Comment}

This is the first large-scale prospective study for long-term safety and tolerability of tolterodine extended release, showing mostly mild side effects and $65 \%$ of the patients completing the entire 12 month treatment period. Few long-term drug studies are performed in children, which makes this study more significant. My regret for the study is that they did not include an efficacy arm so that a practitioner could have all the information necessary to make wise treatment choices for their patients that may need long-term care.

Dr. Brent W. Snow

Division of Urology

University of Utah Health Sci Ctr

Salt Lake City, Utah, USA

E-mail: brent.snow@hsc.utah.edu 